\title{
Discursive design thinking: The role of explicit knowledge in creative architectural design reasoning
}

\author{
KINDA AL-SAYED, ${ }^{1}$ RUTH CONROY DALTON, ${ }^{1}$ AND CHRISTOPH HÖLSCHER ${ }^{2}$ \\ ${ }^{1}$ Bartlett School of Graduate Studies, University College London, London, United Kingdom \\ ${ }^{2}$ Center for Cognitive Science, University of Freiburg, Freiburg, Germany
}

(Received April 15, 2009; ACCePTED October 15, 2009)

\begin{abstract}
The main hypothesis investigated in this paper is based upon the suggestion that the discursive reasoning in architecture supported by an explicit knowledge of spatial configurations can enhance both design productivity and the intelligibility of design solutions. The study consists of an examination of an architect's performance while solving intuitively a well-defined problem followed by an analysis of the spatial structure of their design solutions. One group of architects will attempt to solve the design problem logically, rationalizing their design decisions by implementing their explicit knowledge of spatial configurations. The other group will use an implicit form of such knowledge arising from their architectural education to reason about their design acts. An integrated model of protocol analysis combining linkography and macroscopic coding is used to analyze the design processes. The resulting design outcomes will be evaluated quantitatively in terms of their spatial configurations. The analysis appears to show that an explicit knowledge of the rules of spatial configurations, as possessed by the first group of architects can partially enhance their function-driven judgment producing permeable and well-structured spaces. These findings are particularly significant as they imply that an explicit rather than an implicit knowledge of the fundamental rules that make a layout possible can lead to a considerable improvement in both the design process and product. This suggests that by externalizing the design knowledge and restructuring it in a design model, creative thought can efficiently be evolved and stimulated.
\end{abstract}

Keywords: Design; Discursive; Linkography; Macroscopic Analysis; Space Syntax

\section{INTRODUCTION}

Whether the techniques implemented in problem solving are discursive $^{1}$ (marked by explicable arguments and analytical reasoning) or nondiscursive (driven by intuition), the role of explicit architectural knowledge is fundamental to understand the process of design. Explicit knowledge can be demonstrated when an individual clearly presents knowledge of a fact while being fully conscious and aware of this knowledge. Implicit knowledge of a phenomenon can be identified when the individual represents his/her knowledge by merely reflecting the features of a phenomenon without stating a deep understanding of its core properties. These two definitions are in accordance with a study made by Dienes and Perner (1999). They formulated these definitions through experi-

Reprint requests to: Kinda Al-Sayed, 10 Hanno Close, Wallington, Surrey SM6 9BZ, UK. E-mail: k.sayed@ucl.ac.uk

${ }^{1}$ Discursive thinking is "a conscious process that can be communicated and influenced. Facts and relationships are consciously analysed, varied, combined in new ways, checked, rejected, and considered further ... This type of thinking involves checking exact and scientific knowledge and building this into a knowledge structure" (Pahl \& Beitz, 1996, pp. 48-49). ments on natural language and the behavior of people with and without knowledge of the rules of grammar. This study is based upon an analogous approach. However, in this research the material of study is (built on architectural) space as a "morphic language" that has an underlying structure. A morphic language, according to Hillier and Hanson (1984), is "any set of entities that are ordered into different arrangements by a syntax so as to constitute social knowables." This research will compare implicit versus explicit forms of knowledge of spatial structure: an implicit form that is analogous to knowledge of natural language, and an explicit form of knowledge created through exposure to the rules and configurational theories of spatial morphology. This research starts from the hypothesis that explicit knowledge can prove to be more efficient than implicit knowledge in raising the productivity of design behavior in architecture and enhancing design solutions and this can be reflected in logical models for design reasoning.

It is fundamental to understand that design in both engineering and architectural disciplines is a creative knowledge-based activity. It is creative in the sense that architecture 
is a cumulative search for optimal solutions and it does so by overcoming conflicting design problems. In a way, the optimum result cannot be inferred directly or instantly from the design question and could be considered the product of a creative activity. This product is a response to the design problem embodied in its material solution; thus, architecture itself is an act of creation and otherwise cannot be considered as architecture (Carrara \& Fioravanti, 2005). The creative act in architecture can be supplemented by the domain of architectural knowledge and what the act implies in terms of elements of spatial structure and social organization instantiated in the complex, yet necessary, association between form and function. This can be further enriched by a sound knowledge of other disciplines that intersect with architecture in relation to the specific problem-solution case presented.

With regard to the problem definition in both architectural and engineering disciplines, the problem definition in architectural design, can be more ambiguous than the one of engineering design, given that design problems in architecture are frequently ill defined. This makes architectural design problems hard to configure and quantify. However, it seems to be hard to distinguish between a "well-structured problem" and an "ill-structured one," according to Simon (1984), who suggested that architects start with global specifications and have, from experience, "some over-all organization, or executive programme" regarding the design process. The design problem is then decomposed hierarchically and transformed through memory from its "ill state" to a well-structured problem. Following the design problem configuration, architects retrieve knowledge while reasoning about their design decisions. They also set constraints according to their background as well as the specific design situation to narrow their design solutions. In this way, architects acquire their domain knowledge that they have in the form of design principles, heuristics, theoretical methods of design generation, and similar design experiences to the case presented in the design problem (Maher et al., 1995).

Architects face design problems by recalling the hermeneutical process of reasoning in which they retrieve and reframe the within-domain and cross-domain knowledge they store in memory. They reason about their design decisions through matching the current problem they face with similar situations in their past experiences. This happens throughout the course of analogical reasoning (Carbonell, 1983, 1986) starting from identifying the design problem, followed by reviewing related experiences and selecting the most relevant to be the main focus. As a consequence, knowledge retrieved from memory can be matched to the design situation resulting in a transformation of the design state. Carbonell accounts of analogical reasoning in addition to similar artificial intelligence approaches have been adapted by Oxman (1990) to be applied to architectural design process. According to her, analogical reasoning is a process of matching between the source domain knowledge embedded in the designer's memory in the form of relevant abstract concepts and the target domain knowledge presented in the design situation. An- alogical reasoning is one of the crucial cognitive mechanisms that characterizes creative thinking. It occurs as a consequence of a higher order activity, namely, mental visual imagery as Goldschmidt (2001) has revealed. Imagistic reasoning enables a designer to distinguish different typology.

Both analogical and imagistic mechanisms are instrumental in problem solving and take action within the conceptual level of mental activity. Kryssanov et al. (2001) provide a reading of the sequential conceptual procedures taking action during the analogical reasoning process. In their model, analogical (metaphorical) reasoning passes through four phases. During the first phase, the search for potential solutions is facilitated by matching between the source domain and the target domain in an approach analogous to Oxman's (1990). This matching marks the second phase after which a blending of conceptual spaces in both domains takes effect. In the fourth phase, the design situation is reinterpreted and transformed according to the resulting knowledge. This model offers a comprehensive demystification of the reasoning process. Yet, no clue is provided to indicate the timing of the reasoning process whether before or after the design decision. It is ambiguous whether this reasoning process takes place before finding solutions or whether architects build conjectures about possible design solutions and follow that by rationalizing their design proposals. Nonetheless, it is crucial to our understanding that knowledge is exploited in each part of the design process to create connections between original ideas. Both the novelty and diversity of such connections characterize creative design thinking. Original ideas can be characterized as new, valuable, and novel ideas produced by the individual architects during the design session. The definition of original ideas in this research corresponds to "psychological creativity" identified by Boden (1994), and is not related to her description of "historical creativity." She refers to psychological creativity when the ideas produced by the individual are new to him or her. It does not matter whether these particular ideas have occurred to others before that. Historical creativity in Boden's definition takes place when the ideas produced by the individual have never occurred to others before.

Architectural knowledge is what makes creative design efficient and responsive toward the design problem. It does so by subjecting design solutions to different stages of optimization that take place because of the architect's perceptual and mental activity. The design optimization stages are based upon implementing constrains in filtering design ideas. According to Hillier (1996), they are categorized in three filtering processes that take effect on different levels. The first stage of design optimization is characterized by filtering design ideas according to a fundamental set of constrains that transform design solutions from the state of probability to the state of possibility. The second stage of optimization takes effect through further filtering design solutions using a second set of selected constrains, which, although playing a role for defining the particularities of the design outlook, is less essential than the first stage in setting the configurations 
of space and more dependent on the architect's personal interests. The third stage of filtering is fully dependent on the architect's idiosyncrasies and personal background. The first stage of design optimization is explained as the "generic function" in studies made by Hillier (1996). He identifies the generic function as the simple logic of occupation and permeability in space. On the whole, knowledge of the generic function is embedded in architectural design education and practice. The principles of space syntax extensively explained by Hillier and Hanson (1984) and by Hillier (1996) have exposed some of this knowledge through a systematic analysis of the spatial configurations of the built environment. In our approach we will concentrate mainly on the first set of filters in restricting design solutions, namely, the set of constraints that identify the generic function of space.

Generic function is concerned with the simple functions of occupation and circulation in space as well as its visual configurations and what these functions imply in terms of the permeability and intelligibility of the built environment. In other words, all what makes a design possible. Such a "spatial logic" was gradually formulated by space syntax researchers over the past three decades (http://www.spacesyntax.net/ symposia/index.htm). As a result of their findings, mathematical rules were extracted from the spatial and visual configurations of space to measure the attributes of spatial structures quantitatively. These rules, mostly based on network theory, have identified important relations between spaces in terms of adjacency, accessibility, and visibility. The rules were found to show often remarkable correspondence with the actual social behavior in space. Therefore, these rules were implemented in syntactic methods of spatial analysis that form the instrumental parts of space syntax theory. The familiarity with these methods and the logic they represent, as Hillier (1996) argues, may play part in the architectural design process through achieving a better understanding of the real built environment. Hillier's argument can only be valid provided the evidence that these methods are effective in spatial analysis. This condition was verified by the observed correspondence between spatial form and social behavior. A deeper understanding of the structural and organizational logic of space provided by space syntax theory can lead to the possession of an explicit form of this knowledge. This knowledge can then feed into the act of design through subjecting designs to a type of process of logical reasoning. This study contributes to both space syntax research and cognitive science research. It does so by presenting one of the first attempts to investigate how the explicit knowledge of spatial configurations in general and of generic function in particular can positively influence architects during the act of design. This positive effect affects design-process productivity and the attributes of the spatial and visual configurations of the design solution.

In this research, it is hypothesized that space syntax methods are not only effective in terms of evaluating existing spatial structures but can also support architectural design thinking by evaluating design ideas throughout a design's evolution, thus generating enhanced spatial configurations.
Space syntax measures usually play a feedback role in a computerized design process to evaluate ready-made design solutions. This study is mainly concerned with a possible generative role in which knowledge of syntactic measures might serve during intuitive problem solving in architecture. The discursive design process is therefore based on explicit knowledge of spatial configurations (space syntax) and implies the use of logic and systematic reasoning rather than intuition in problem solving. This paper will attempt to provide evidence that this form of discursive design can prove to be more efficient than the nondiscursive one in creating improved spatial configurations (Hillier, 1996). ${ }^{2}$ Space syntax is an architectural theory in which certain spatial elements are considered as nodes in a network. These nodes are believed to be connected if there is the ability to move between them (they are spatially connected). The "depth" of spatial elements in a network can be computed through a set of mathematical operations. These mathematical operations are normally calculated using computer techniques and are hard to predict intuitively. However, this paper suggests that a basic implementation of syntactic rules can actually support the intuitive design processes. Whether this is actually the case will be investigated in this study to scrutinize the capacity of space syntax as an effective evaluative tool for thinking about architectural design.

To investigate the role of explicit knowledge in architectural design, a design task will be presented to two groups of architects asking them to solve a well-defined yet sufficiently open problem. The first group will have implicit knowledge of spatial configurations, and it is therefore hypothesized that they will attempt to solve the design problem using nondiscursive methods. The other group will have an explicit knowledge of spatial configurations and should hypothetically be more likely to use discursive techniques in their design decisions. Cognitive and spatial analyses will be then applied to the design solutions to detect differences and regularities between the design performance and outcomes of both groups of architects. Cognitive analysis will basically combine different protocol analysis approaches. Spatial analysis will simply be an implementation of space syntax quantitative evaluation techniques.

\section{PILOT STUDY}

The pilot study is designed in a form of an intentionally limited design task to be solved intuitively by the participants who share a particular domain knowledge limited to the field of architecture. This means that participants should have special-

\footnotetext{
2 "An architectural theory is an attempt to render one or other of the nondiscursive aspects of architecture discursive, by describing non-discursivity in concepts, words, and numbers. We may say that an architectural theory seeks to create a 'nondiscursive technique', that is, a technique for handling those matters of pattern and configuration of form and space that we find it hard to talk about. In research terms we could say that an architectural theory, at least in the 'narrow' aspects through which it describes and prescribes design decisions, is an attempt to control the architectural variable." (Hillier 1996, p. 59)
} 
ized knowledge in architecture provided that all of them have worked as architects in design practices at some stage in their career. However, it is very hard to have participants of equivalent backgrounds in each group because individual differences will always arise. In addition, a designer's experience in architecture can influence his/her design approach and idiosyncrasies, but also a designer's cultural background and life experiences can play a role in shaping his/her thoughts during a design process. It is also important to understand that architectural knowledge needs to be wide ranging and interdisciplinary for a design solution to cover all the issues raised by a design problem (for a discussion of such multiconstraint requirements, see Bertel et al., 2007); participants therefore are expected to have different architectural experiences and backgrounds according to the different educational methods and design projects they have been subjected to. Yet, a previous experience in architectural design is one of the basic requirements of the subject selection criteria. The participants in the first group have additional knowledge in the form of theoretical and practical experiences using space syntax methodology. It is further required that architects in the first group have basic theoretical understanding of what space syntax theory implies and its applications in architectural and urban design. In contrast, in the second group, participants share a general architectural education and no specialist knowledge of space syntax theory. However, they do have a form of implicit knowledge of spatial configurations; they can provide simple descriptions for spatial representations and general spatial structure but they do not have a conscious knowledge of the discrete structure of space. In other words, they do not have the theoretical knowledge with which they would be able to identify and retrieve attributes from a spatial structure in the form of morphological rules.

A comparative analysis of the performance of both groups will be conducted by evaluating their design proposals to highlight any commonalities and differences between the groups. The study will start investigating the influence of explicit versus implicit knowledge of spatial configurations on architects' design processes through subjecting their semantic explanation of their design actions to a combined model of protocol analysis. Their design solutions will then be evaluated in terms of the quality of social organization that their designs promote, and in terms of the quantitative attributes of their designs' spatial configurations.

To observe the role of space-configurational knowledge in driving design decisions, a set of interviews was organized with 12 architects. Half of them had either an academic or practical background in space syntax methods/theory in addition to their original architectural background. We refer to these as the "SSX group." The remaining six architects, the "NSSX group," had traditional, architectural design backgrounds, and no familiarity with space syntax theory. The first part of the interview started with a set of questions about the architect's experience and general design process strategy, particularly in terms of allocating occupational spaces and organizing the circulation within a layout (see Table 1). In the second part of the interview, architects were given a design brief for an architect's office floor plan and were asked to accommodate required functions within a predefined layout ${ }^{3}$ while taking into account adequate circulation space (see Table 2). The layout was intentionally restricted to one level rather than multiple levels to simplify the design problem, and hence, the design solutions can be limited to two-dimensional rather than three-dimensional visualizations. In this way, the participants were constrained to concentrate on the spatial configurations in a planar setting. The layout was a hypothetical rectangular floor plan in a skyscraper with two access points from two cores. Although the second phase was limited to $15 \mathrm{~min}$, most of the participants exceeded the specified time period to complete the task. The brief was for the design of an architect's office because this would be an extremely familiar space to any architect given that he or she would have had at least some experience working in an architectural practice. Therefore, it is expected that the participants have an experience of, at the very least, working in a design office, even if they have not any previous experience of designing such an office layout.

The brief and the layout together define the design problem and present the first set of constraints that will help to narrow the range of possible design solutions. The rationale behind narrowing the design task to a simple allocation of function together with defining circulation was to indicate to the designer, acts associated with the first set of optimizations that are hypothesized to take place during a design process, namely, those of "generic function" (Hillier, 1996). The task in general was intended to be easy to follow. The simplicity of the design task was later remarked upon by 6 out of 12 participants from both groups. They mainly complained that the task was too simplified and entailed hardly any complex mental challenges. Architects were asked to comment upon their design process while externalizing their ideas on tracing paper, overlaid on the original floor plan. In this way they were constrained to solve the design problem intuitively using sketches without any access to a computer. Sketching in design is one of the most efficient techniques to externalize design ideas in the form of visual representations (Goel, 1995; Oxman, 1997). A video camera recorded the drawing process and a microphone recorded the architect's voice while describing his/her thoughts during the design process. The verbal comments were later transcribed to subject them to protocol analysis. The protocol analysis took into consideration the semantic expressions without including the physical acts themselves. Only the final design outcomes were subject to additional quantitative evaluation. Four females and eight males participated in these experiments. Their academic plus practical experiences in architecture ranged between 10 years and 21 years. The ultimate time taken by the participants to solve the design tasks ranged between 11 and $50 \mathrm{~min}$.

\footnotetext{
${ }^{3}$ The layout is cited in Shpuza (2006, pp. 164, 315-316), and it is an existing one belonging to Weyerhaeuser Company SOM (Sidney Rodgers \& Associates Tacoma, WA).
} 
Table 1. Participant experiences and interests

\begin{tabular}{|c|c|c|c|c|}
\hline Architects & Experience $^{\mathrm{a}}$ & $\begin{array}{l}\text { Task } \\
\text { Period }\end{array}$ & $\begin{array}{l}\text { Word } \\
\text { Counts }\end{array}$ & $\begin{array}{c}\text { Architect Thoughts About Spatial Permeability } \\
\text { During Design Process }\end{array}$ \\
\hline \multicolumn{5}{|c|}{ NSSX Group } \\
\hline $\mathrm{AH}$ & 17 & $18 \mathrm{~min}$ & 1685 & I always try to optimize circulation in relation to occupation. \\
\hline $\mathrm{LC}$ & 20 & $49 \mathrm{~min}$ & 5426 & $\begin{array}{l}\text { Thinking about the logic of circulation and occupation; this spatial } \\
\text { structure is hierarchical reflecting the organization. }\end{array}$ \\
\hline $\mathrm{AB}$ & 13 & $38 \mathrm{~min}$ & 3132 & $\begin{array}{l}\text { Depending on the scale, scope of the project, client demands, and how } \\
\text { functions determine circulation }\end{array}$ \\
\hline KS & 11 & $32 \mathrm{~min}$ & 1189 & Connecting main spaces visually; circulation is a secondary issue. \\
\hline $\mathrm{OO}$ & 21 & $38 \mathrm{~min}$ & 2673 & On the basis of client requirements, the context, and the environment \\
\hline JG & 11 & $11 \mathrm{~min}$ & 651 & Depending on the design program \\
\hline \multicolumn{5}{|c|}{ SSX Group } \\
\hline $\mathrm{BC}$ & 11 & $15 \mathrm{~min}$ & 1191 & Circulation is a result of making connections between static spaces. \\
\hline PE & 11 & $27 \mathrm{~min}$ & 2305 & Setting the organizational structure and grouping functions \\
\hline AN & 11 & $18.5 \mathrm{~min}$ & 1118 & Thinking about the client, the users, and functional organization \\
\hline $\mathrm{CE}$ & 10 & $30.5 \mathrm{~min}$ & 1374 & $\begin{array}{l}\text { Space, connections, and circulation are basic layers of the design } \\
\text { process; the top layer is an ideal one. }\end{array}$ \\
\hline $\mathrm{CR}$ & 19 & $15 \mathrm{~min}$ & 1819 & - \\
\hline LM & 13 & $16.5 \mathrm{~min}$ & 770 & Depending on the functions \\
\hline
\end{tabular}

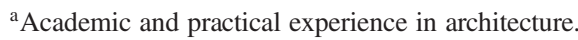

\section{PROTOCOL ANALYSIS OF DESIGN PROCESSES}

Previous research approaches toward decoding the design process have concentrated either on the strategy of design performance or on design content. The methods of analysis that will be implemented in this article focus on content while also taking into account the protocol analysis (Ericsson \& Simon, 1993).

The present study concentrated on evaluating the design performance of architects and the configurations of their design products (the resulting layouts). Our design task matches real design situations that are in their nature quite intricate and require complex cognitive activity that takes place on different levels. Design, as we will see later in this paper, is a nonlinear process that involves feedback loops. These loops happen as architects explore new ideas and retrieve older ones throughout the design process. This complex path of idea production and reproduction can be represented in Goldschmidt's linkograph model (1990, 1992). This particular model will be used in combination with a detailed model of cognitive coding as an integrated protocol analysis method to analyze design performance in our current investigation. The cognitive coding model is based on the macroscopic analysis of designer's cognitive actions proposed by Suwa et al. (1998). It will be used to code the linkography model as a supplementary fine-grained layer to further indicate what kind of cognitive activity is mostly associated with critical design moves and whether certain activities are more highly connected in the linkograph network. In this way, design performance will be subjected to a joint model of protocol analysis including both linkography and macroscopic analyses methods.

In Goldschmidt's scheme the protocol is segmented to a set of "design moves" with directed links. A design move is explained as "an act of reasoning that presents a coherent pro-

Table 2. Design task including a brief for an architect's office and an existing layout

\begin{tabular}{l}
\multicolumn{1}{c}{ Design Brief } \\
- Head office with its private secretary space \\
- Waiting area with small exhibition \\
- Two meeting rooms \\
- Management offices (3-4) \\
- Telecommunication offices (2) \\
- Three spaces for consultants \\
- Spaces for five project directors, each with two associates, \\
and design team \\
- Two IT offices \\
- Two technical studies units \\
- Two construction expertise unit \\
- Twice areas with small kitchen, toilets, and lounge
\end{tabular}


position pertaining to an entity that is being designed" (Goldschmidt, 1992). Links among moves, which are determined by the researchers, are notated in a network. The design process can then be interpreted as a pattern of links among moves associated in the graphic network of the "linkograph." Design moves according to Goldschmidt are interconnected with two kinds of directed links: links connecting to previous moves are identified as "backlinks" and links connecting to subsequent moves are identified as "forelinks." In Goldschmidt's (1995) definition: "The two kinds of links are very different conceptually: backlinks record the path that led to a move's generation, while forelinks bear evidence to its contribution to the production of further moves." Goldschmidt (1990, 1992) found a high correlation between design productivity and the ratio of links/moves, which she identified as the link index. She also recognized that moves that have a dense linkage connection, both forward and backward, namely, critical moves (CMs), can be further evidence of design productivity. The trend of sequential, critical moves represents the critical path of a design process. Each design move involves retrieval of knowledge in a process of reasoning. Linking different design moves represents structuring and reframing the knowledge recalled into the design problem-solving process. This process of knowledge selection, retrieval, blending, and restructuring marks creative thought.

The second approach used in this research is a contentbased analysis model proposed by Suwa et al. (1998). It is intended to integrate with the linkography model. In their method, Suwa et al. (1998) provided a macroscopic representation of the design process by segmenting the cognitive actions extracted from the designer's physical actions and semantic expressions. The analysis in this paper will be limited to the semantic data because of the complexity and length of the design processes at hand. Unlike Suwa et. al.'s (1998) approach to record the semantic comments of designers by the end of their design process, the semantic expressions will be recorded during the design process itself. This is mainly justified by the fact that a final design solution does not always represent the entire process of thinking. Apart from this, architects may postrationalize their design acts after arriving at an endpoint with their design solutions. The categorizations model of Suwa et al. (1998) is documented in Table 3, and provides a detailed account of the categorization's nature and criteria. Their description separates physical, perceptual, functional, and conceptual cognitive actions. They have also subcategorized the physical and conceptual actions. It must be emphasized that their categorization model was implemented only partially in the paper because of the different scope of this research, which deals with discursive thinking in architecture. Suwa et al.'s (1998) scope was mainly about proposing a very fine-grained level of labeling into the design process analysis by addressing the cognitive activity of designers in action. They also recorded the physical activity during the drawing process. The physical activity was ignored in this paper because of the fact that it cannot be interpreted semantically by designers; hence, the clue provided by tracking the physical activity does not offer a clear indication about the knowledge embedded in the designers' minds. The only physical action that is taken into consideration in this paper is the look action (L-action), which represents the state when designers look at previous depictions and refer to them semantically. The perceptual, functional, and conceptual actions will be fully considered as long as they are expressed in the architect's statements. Perceptual actions (P-actions) will be recorded whenever the architect refers to visual features or spatial relations. Functional actions (F-actions) occur when the architect considers interactions between artefacts and people/nature as well as when he thinks about the psychological reactions of people. Conceptual actions may occur during the process of knowledge retrieval (K-action), or whenever the architect makes preferential and aesthetical judgments (E-action), or when the architect defines a goal (G-action). The original segmentation model of Suwa et al. (1998) regards every segment according to a corresponding reference. For instance, talking about building cores defines one segment, and talking

Table 3. Cognitive actions categorization model

\begin{tabular}{|c|c|c|c|}
\hline Category & Names & Description & Examples \\
\hline \multirow[t]{3}{*}{ Physical } & D-action & Make depictions & Lines, circles, arrows, and words \\
\hline & L-action & Look at previous depictions & - \\
\hline & M-action & Other physical actions & Move a pen, move elements \\
\hline \multirow[t]{3}{*}{ Perceptual } & P-action & Attend to visual features of elements & Shapes, sizes, and textures \\
\hline & & Attend to spatial relations among elements & Proximity, alignment, and intersection \\
\hline & & Organize or compare elements & Grouping, similarity, and contrast \\
\hline \multirow[t]{2}{*}{ Functional } & F-action & $\begin{array}{l}\text { Explore the issues of interactions between artifacts } \\
\text { and people/nature }\end{array}$ & $\begin{array}{l}\text { Functions, circulation of people, views, and } \\
\text { lighting conditions }\end{array}$ \\
\hline & & Consider psychological reactions of people & Fascination, motivation, and cheerfulness \\
\hline \multirow[t]{3}{*}{ Conceptual } & E-action & Make preferential and aesthetic evaluations & Like-dislike, good-bad, and beautiful-ugly \\
\hline & G-action & Set up goals & - \\
\hline & K-action & Retrieve knowledge & - \\
\hline
\end{tabular}

Adapted from M. Suwa, T. Purcell, and J. Gero, Macroscopic analysis of design processes based on a scheme for coding designers cognitive actions, 1998, Design Studies 19(4), p. 460. Copyright 1998 Elsevier. Adapted with permission. 
about design teams defines another segment. Further detailed segmentations then refer to the different cognitive actions whether physical, perceptual, functional, or conceptual. In our case, the categorization model of cognitive actions will be used to code the design moves in Goldschmidt's (1992) linkographs rather than the design segments proposed by Suwa et al. (1998). An example of the cognitive actions coding in a linkograph is represented in Figure 1. In part of this example (moves 12 to 17) the segmentation of design moves and the associated model of coding is as the following:

12. "... this is in general the first impression about what I can see now." (E-action)

13. "Now I guess I need to study first the areas. .." (G-action)

14. ". . . because I believe that this is about 30 meters width and maybe 90 or 80 length for the space and I wish I can know the height of the space." (P-action)

15. “. . . because I need to understand if I can design this space as a multilevel space. .." (F-action)

16. "ok I will consider it as a flat one floor." (P-action)

17. "I think according to the new theory for the working environments. .." (K-action)
As can be seen in Figure 1 the design move number 13 is linked to number 14 , number 14 is linked to number 15 , and number 15 is linked to the design move number 16 . The design move number 12 is linked to all the moves before it. The design move number 17 is linked to five subsequent moves. The coding represents another layer of segmentation to be added to the linkograph to reveal which kinds of cognitive actions are more productive and more likely to be associated with the critical moves. To detect regularities and distinctions in the data of the semantic transcripts, the linkography models of all design processes will be examined in terms of productivity and statistical measures. The cognitive coding of the linkographs may then reveal further information concerning the cognitive nature of the design moves.

\subsection{Linkography analysis}

Linkography analysis will take into account the productivity measures defined by Goldschmidt (1990, 1992, 1995) as well as a set of statistical descriptions of the linkographs data (Kan $\&$ Gero, 2008). Productivity measures include the link index and the critical moves. The link index is obtained by normalizing the number of links against the number of moves as de-

\section{Cognitive coding $\mathrm{CM}^{8}$ backlinks $\mathrm{CM}^{8}$ forelinks}

\section{Design moves}
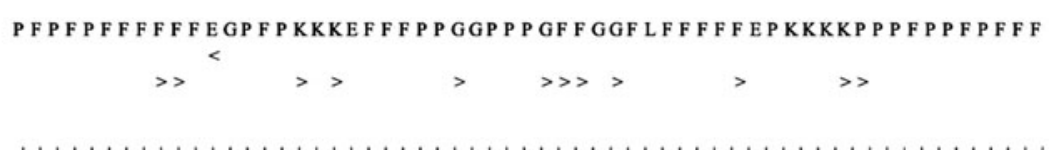

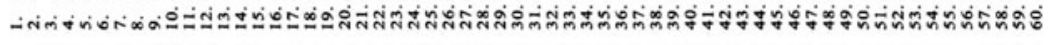

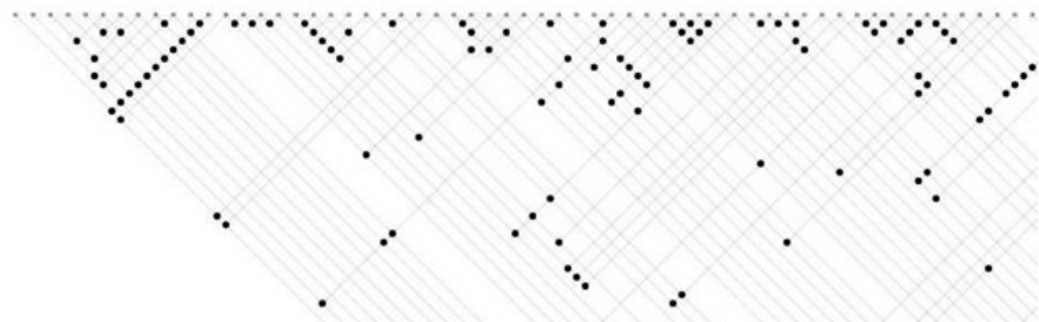

- $\quad$ Nodes

- Links

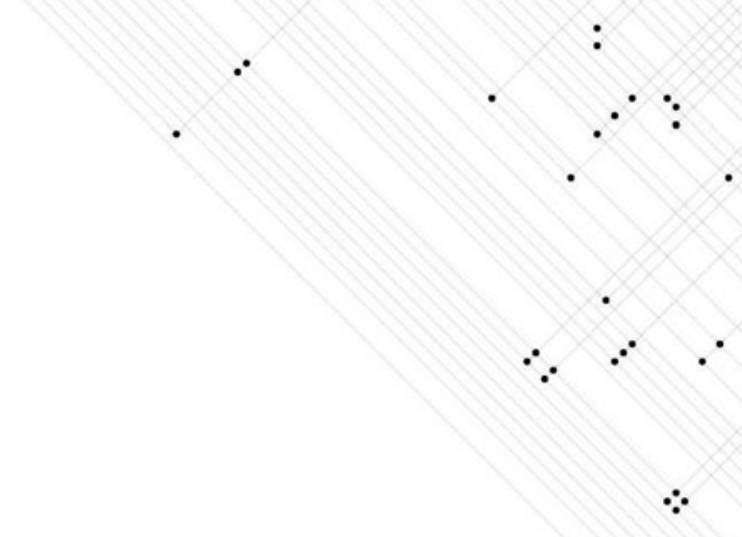

Fig. 1. Coding the design moves in a linkograph according to the macroscopic cognitive model proposed by Suwa et al. (1998). 
Table 4. Link index and critical moves values of linkographs

\begin{tabular}{|c|c|c|c|c|c|c|c|c|c|c|c|c|}
\hline \multirow[b]{3}{*}{ Participants } & \multicolumn{12}{|c|}{ Groups } \\
\hline & \multicolumn{6}{|c|}{ NSSX } & \multicolumn{6}{|c|}{ SSX } \\
\hline & $\mathrm{AH}$ & $\mathrm{LC}$ & $\mathrm{AB}$ & $\mathrm{KS}$ & $\mathrm{OO}$ & $\mathrm{JG}$ & $\mathrm{BC}$ & $\mathrm{PE}$ & AN & CE & CR & $\mathrm{LM}$ \\
\hline Link index & 3.17 & 4.97 & 2.82 & 2.6 & 4.75 & 3.31 & 3.97 & 6.42 & 2.57 & 3.61 & 3.12 & 2.53 \\
\hline \multicolumn{13}{|l|}{$\mathrm{CM}^{8}$} \\
\hline Backlinks & 13 & 37 & 12 & 6 & 31 & 7 & 12 & 55 & 5 & 16 & 6 & 4 \\
\hline Forelinks & 12 & 45 & 17 & 6 & 35 & 6 & 11 & 60 & 3 & 15 & 14 & 4 \\
\hline Total & 25 & 82 & 29 & 12 & 66 & 13 & 23 & 115 & 8 & 31 & 20 & 8 \\
\hline
\end{tabular}

scribed by Goldschmidt (1995). Critical moves in her definition are moves that have high number of links. In our case, we consider the critical moves that have more than eight moves, which we refer to as $\mathrm{CM}^{8}$.

The link index (see Table 4) varies between the participants in both groups. The average value of all link index values in the SSX group (3.7) is slightly higher than the one in the NSSX group (3.6). There are significant differences on the individual level between the participants. One architect in the SSX group seems to have an exceptionally high link index (6.42). This participant is PE who is the only member in SSX group who has working experience rather than theoretical knowledge of space syntax theory and methods. It is also apparent in Table 4 and Figure 2 that this particular participant has the highest count and percentage of critical moves among all other participants. This is considering the critical moves that have more than eight links. Provided that the linkographs have different numbers of design moves, percentages of design moves are more comparable than their corresponding direct counts. The percentages of critical design moves $\left(\mathrm{CM}^{8}\right)$ vary broadly within each group. The average value of these percentages illustrated in Figure 2 seems to be slightly higher in the SSX group compared to the NSSX group, but it is not statistically reliable given the small number of participants in this experiment. For example, the average value of the percentages of total critical moves, including backlinks and forelinks, is 23.75 for the SSX group and 21.48 for the NSSX group. Individual differences are more pronounced than the differences between the groups, particularly within the SSX group. PE, CE, and BC from the SSX group along with LC and OO from the NSSX group have relatively high link index values as well as high percentages of $\mathrm{CM}^{8}$ that, according to Goldschmidt (1995), indicates that their designs are highly productive. AN and LM from the SSX group as well as $\mathrm{KS}$ and $\mathrm{AB}$ from the NSSX group have low link index and $\mathrm{CM}^{8}$ percentage values. This may mean that their design performance is the least productive if we apply Goldschmidt's model. Whether this has a corresponding effect on the design solutions of the participants will be investigated the spatial evaluation section of the analysis.

The statistical description of the linkograph is obtained by substituting the linking lines in a linkograph with the nodes that represent their intersections, excluding the design moves themselves. In this way we obtain nodes in a two-dimensional space. By constraining these nodes to an $X$ and $Y$ coordinates, we treat each node as a point with $X$ and $Y$ values. The resulting scheme can be described statistically in terms of the mean values of $X$ and $Y$, that is, the average position of all the nodes, and their standard deviations in the $X$ and $Y$ axes. According to Kan and Gero's (2008) statistical description of linkographs, by adding all the $X$ coordinates of the nodes and dividing the total by the number of all nodes the mean value of $X$ can be produced, which represents the average location of the nodes in the $X$ axis. A lower mean value of $X$ implies that more nodes appear in the beginning of a design process. This signifies that the designer commences the design process by introducing many ideas and continues by considering unrelated design decisions. A higher mean value of $X$ suggests that nodes are more likely to be present at the end of the design process that leads to the conclusion that ideas converge toward the end of the design

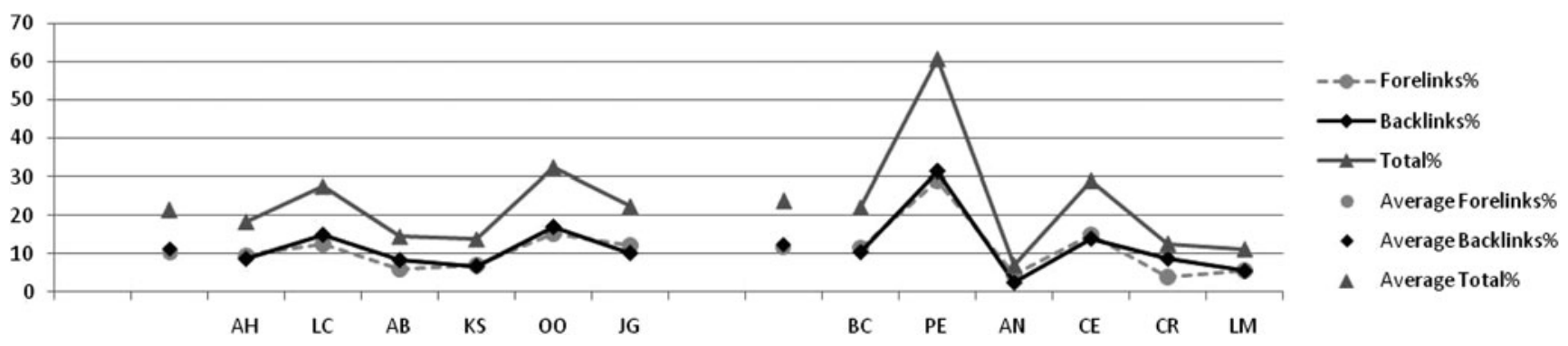

Fig. 2. The critical moves percentages. 


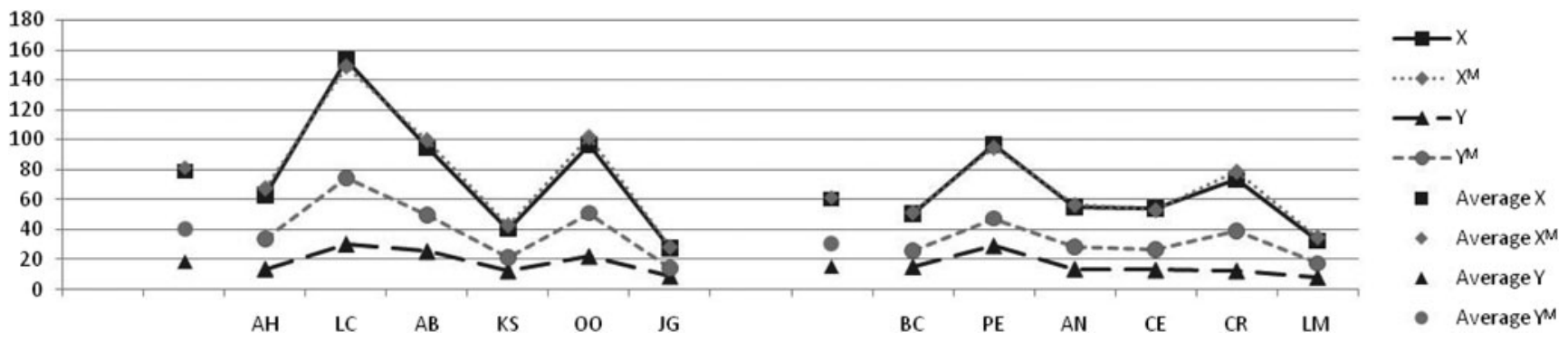

Fig. 3. Mean values of the linkographs.

process. A similar method of calculation is applied to the $Y$ values. Adding up the $Y$ coordinates of the nodes and dividing the result by the total number of nodes will give the mean value of $Y$, which represents the average location of the nodes in the $Y$ axis. A higher mean value of $Y$ indicates longer linking lengths. Given that different linkographs have different counts of design moves, a difficulty seems to be present when comparing the mean values of all the linkographs. For this purpose a method of comparison was introduced to compare each mean value $(X, Y)$ of a linkograph with a corresponding hypothetic mean value $\left(X^{\mathrm{M}}, Y^{\mathrm{M}}\right)$. This hypothetic mean value is calculated considering that all the design moves in a linkograph are interconnected with links. However, the mean values do not indicate how the nodes are dispersed or concentrated around the means. For this reason it is important to measure the standard deviations even though it is hard to compare the standard deviation values in a set of linkographs with different counts of design moves. In general, however, lower values of standard deviation indicate that the nodes are more clustered around the mean.

According to the reviewed methods of statistical analysis, all of the $X$ values of the linkographs are almost equal to their corresponding $X^{\mathrm{M}}$ values (see Figs. 3 and 4). This means that the nodes in each linkograph are basically equally distributed along the $X$ axis. There are no apparent differences between the groups themselves. Unlike $X$ values, differences are notable between $Y$ values and $Y^{\mathrm{M}}$ values in all linkographs. These differences, on average, appear to be more dominant in NSSX case compared to SSX group. The linkographs in SSX case have shorter links connecting closer design moves compared to NSSX case in which there are relatively longer links connecting far-distanced design moves. The standard deviation values indicate how clustered are the nodes around the mean values of the linkographs. Their numeric values as well as their graphical representations are plotted in Figure 4. They can be compared only with caution because of the observed differences in the numbers of design moves.

Goldschmidt (1995) referred to four main types of linkographs. In case 1, design moves are completely unrelated, indicating low potentials for idea development. In case 2, design moves are completely interconnected, hinting at a fully integrated process in which successive ideas may suffer from fixation and lack of diversity. This leaves fewer chances for novel ideas. In case 3, each design move is linked only to its subsequent move. This signifies a progression in the process with not much development in terms of ideas. In Case 4, design moves are partly interrelated indicating a productive design process that provides plenty of opportunities for idea generation and development.

In our case all the moves in the linkographs are interrelated. Case 1 and case 3 hardly exist anywhere in our linkographs. Case 4 is the most dominant in all linkographs with an occasional but partial occurrence of case 2 . The only way to distinguish between case 2 (an interconnected net of moves) and case 4 (interrelated moves) is to suggest a kind of coding that ranges between case 2 where you can find patches of totally integrated moves and case 4 where you have a diverse structure of interrelated moves. To highlight these differences in nodes clustering in both groups a Ward's method cluster analysis is implemented with JMP statistical software to find clusters in the linkographs data. Statistical clustering was previously investigated by Kan and Gero (2008), with the difference that they used a clustering method based on centroids (the mean values of clusters) rather than Ward's, which is based on variations around the mean, as the Ward method is more reliable in tracking approximate clusters than the centroid method. In addition to the Ward method, a nonparametric density estimation feature ${ }^{4}$ was used to distinguish patterns in the nodes' point density. We have introduced the use of this statistical method to represent the density of nodes' clustering that ranges between highly concentrated nodes matching case 2 and diverse distribution of nodes matching case 4 . The resulting diagrams can be seen in Figure 5. The dark spots indicate to states of concentration on particular ideas that correspond to case 2. A content interpretation of the critical moves in relation to what is actually going on in the design process is presented in Figure 6.

On a general level, the patterns of difference between the two groups are not clearcut. More differences can be distinguished on the individual level compared to the group level.

\footnotetext{
${ }^{4}$ Bivariate density estimation is one of the statistical analysis methods in JMP software. It models a smooth surface that describes how dense the data points are at each point in that surface. The plot adds a set of contour lines showing the density. Optionally, the contour lines are quantile contours in $5 \%$ intervals with thicker lines at the $10 \%$ quantile intervals. This means that about $5 \%$ of the points are below the lowest contour, $10 \%$ are below the next contour, and so forth. The highest contour has about $95 \%$ of the points below it.
} 
NSSX

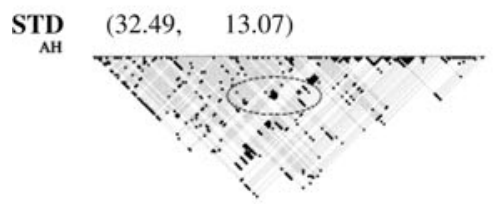

STD

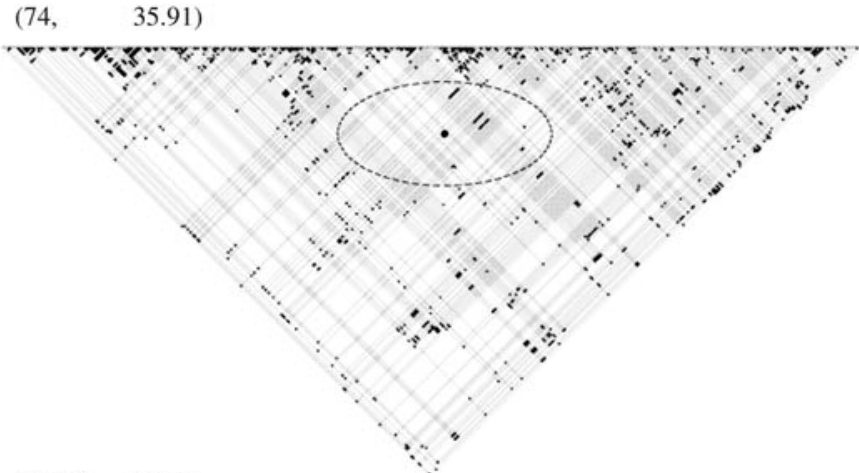

STD $\quad(45.87, \quad 24.69)$

AB

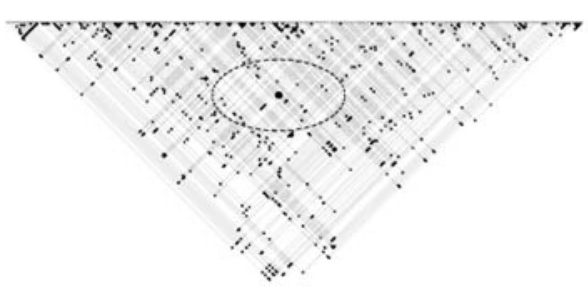

STD $\quad(19.96, \quad 9.97)$

KS

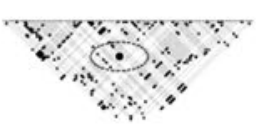

STD $(47.18, \quad 20.3)$

oo

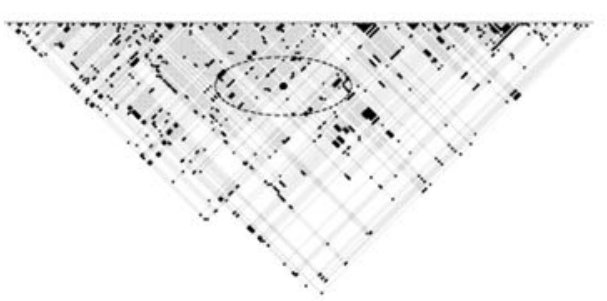

STD (12.26, 7.23)
Jg

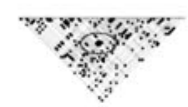

\section{SSX}

BC

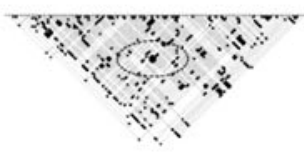

STD

$(24.49, \quad 12.34)$

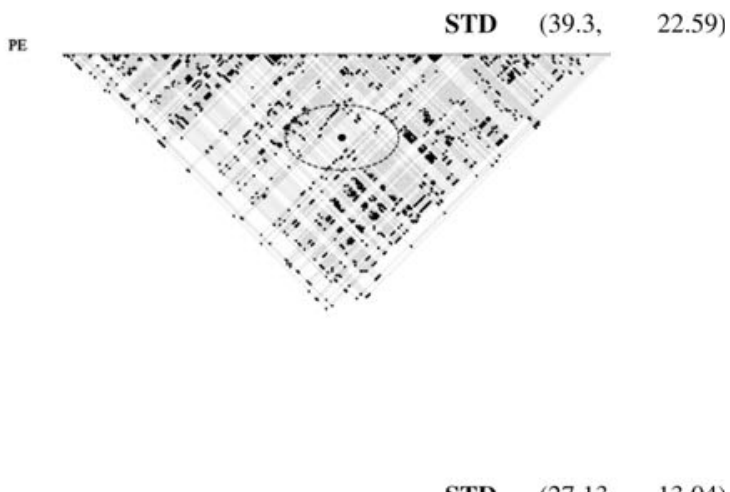

AN

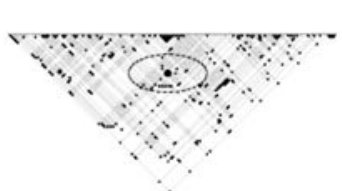

STD $\quad(22.7$,

12.4)

CE

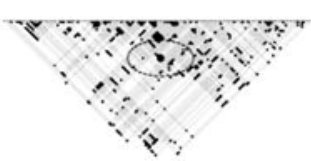

STD $\quad(42.82, \quad 15.05)$

CR

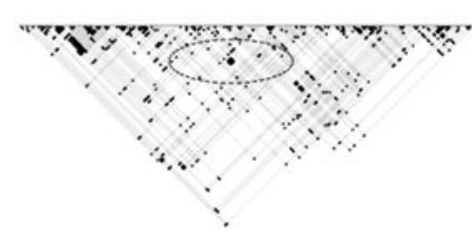

STD

$(17.01$

8.615)

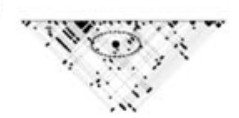

Mean value $(\mathrm{X}, \mathrm{Y})$

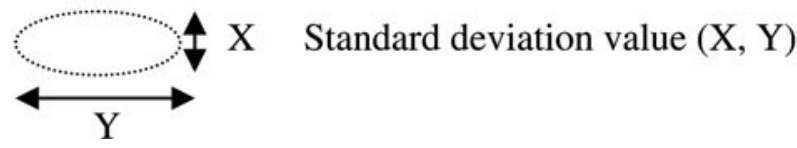

Fig. 4. Linkograph models demonstrating the design processes of all of the participants in the SSX and NSSX groups. The mean and standard deviations of the $X$ and $Y$ values of all nodes are represented graphically on the linkographs. 
2.3.4.6.7.8.9 Quantile Density Contours

NSSX

AH

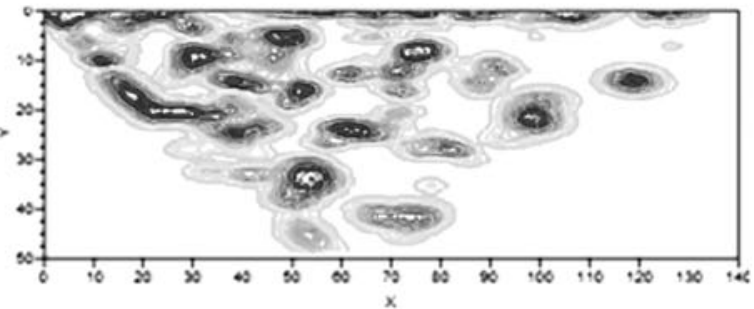

LC

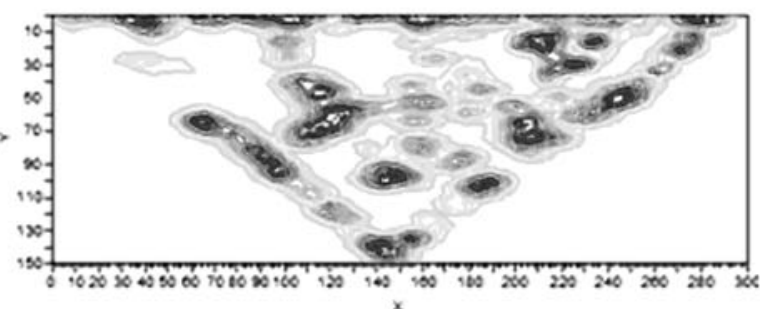

$\mathbf{A B}$

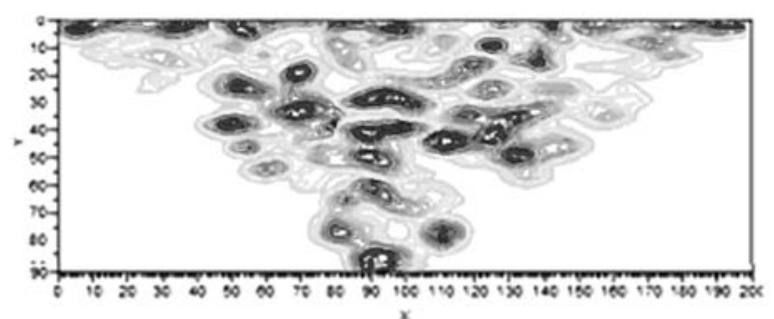

KS

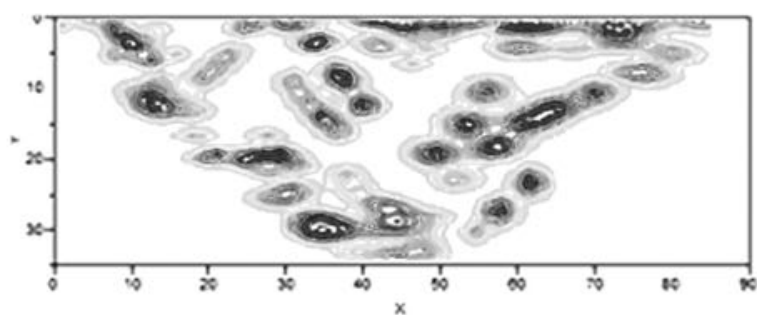

OO
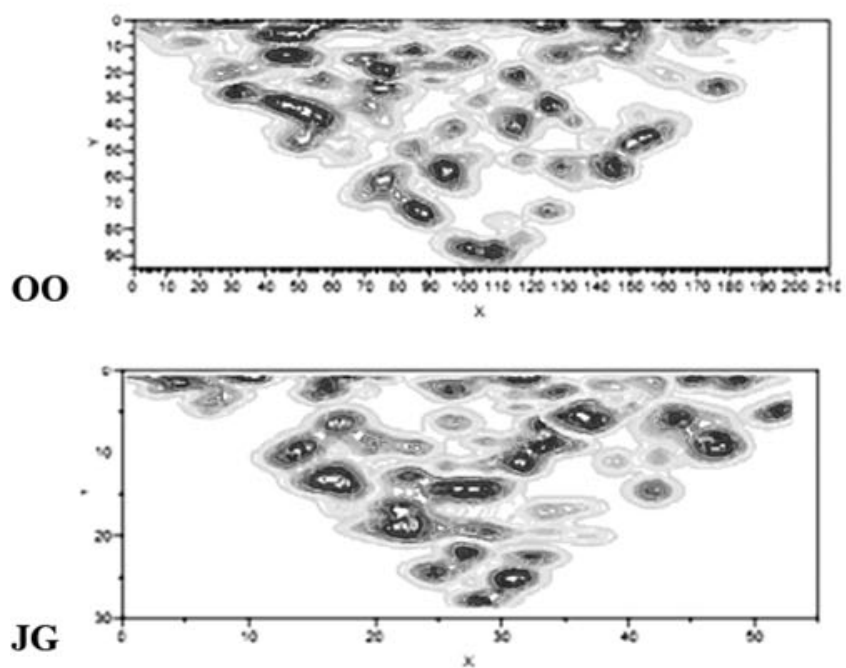

SSX

BC

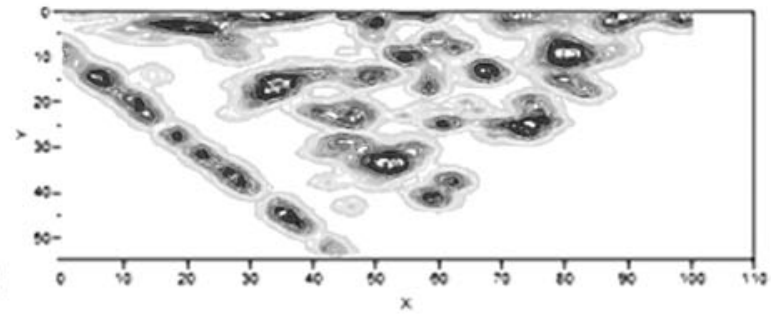

PE

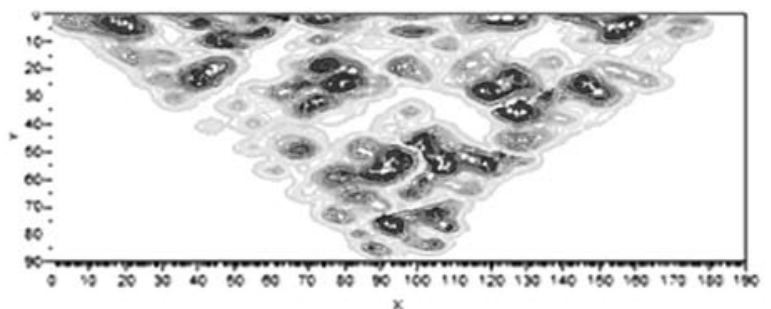

AN

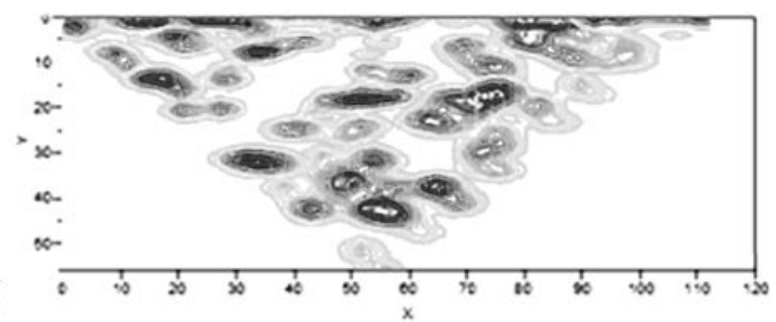

CE
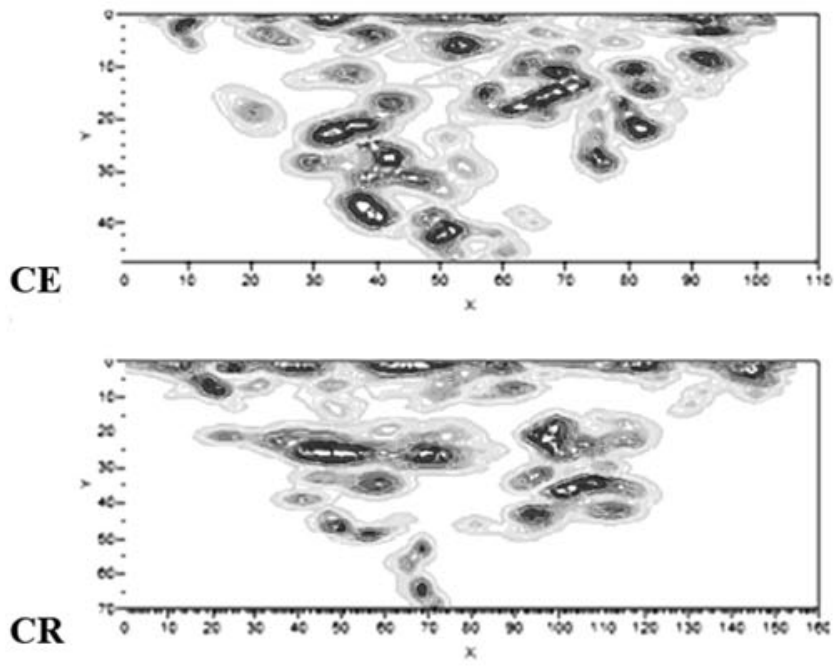

LM

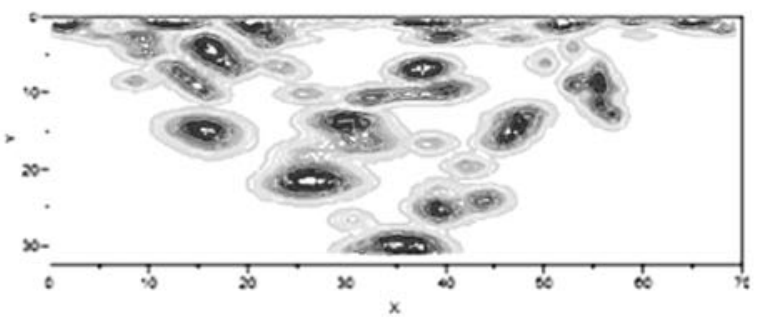

Fig. 5. Nonparametric density estimation of SSX and NSSX linkographs. Diagrams were produced using JMP, Statistical Discovery Software, Version 5.1. 
NSSX

\section{$\mathrm{AH}$}

allocating spaces in the layout

Setting the entrance area

Setting the cconcep

aiming at an open layout

Setting the circulation

Setting a hierarchical structure

allocating spaces

according to the

organizational structure

Setting the cconcept

of the meeting room

Setting the circulation

aiming at an open layou

\section{SSX}

\section{$\mathrm{BC}$}

Categorizing space

adjacencies according

to the entrance area

Categorizing space

according to their public

vs. private character

allocating spaces

Setting the

circulation

allocating spaces

Setting the

circulation

allocating spaces
LC

relating the company

structure to the spatial

arrangement

setting the entrance area

Setting the visual

configurations

allocating the workspace

enclosing meeting space

allocating the workspace

Setting the circulation in

relation to the printer

Setting the cconcept

of the head office

Setting the visual

configurations

allocating spaces according

to the building infrastructure

allocating the lounge area

thinking about space politics

confirming spatial allocations

Setting the circulation

allocating spaces according to the building infrastructure

separating functions

allocating spaces according to the organizational structure

enclosing meeting space

allocating spaces according

to the organizational structure

\section{PE}

Categorizing spaces

according to their public

vs. private character

setting spatial relations

according to the

organizational structure

Setting the entrance area

Setting the circulation

Setting the workspace

allocating spaces

according to the

building infrastructure

Setting the workspace

Setting the entrance area

enclosing meeting space

Setting the circulation

Categorizing workspace

orientation according to

natural light exposure

Setting the workspace

allocating spaces

according to the

organizational structure

Setting the circulation
$\mathrm{AB}$

Setting the

entrance area

aiming at an

open layout

Zoning spaces

Setting the

workspace

Setting the

circulation

allocating spaces

according to the

work culture

Setting the

formal concept

Setting a 3D

visualization

Setting the

workspace

Setting the

entrance area

Setting the

workspace

Setting a 3D

visualization

Setting the

formal concep

Setting the

workspace

\section{AN}

allocating spaces

according to the

organizational

structure

allocating spaces

Setting the cconcept

of the meeting rooms

Setting the
circulation

Setting the

entrance are
KS

Perceive the

layout configurations

Categorizing room

orientation according

to natural light

exposure

Setting the

north direction

applying the

categorizaion rule

Setting the

circulation

perceiving

the scale

Setting the

circulation

applying the

categorizaion rule

Setting the

circulation

(2)

OO

relating the number of

users to the scale of

space specified

aiming at an

open layout

Setting the

entrance area

relating the number of

users to the scale of

space specified

Zoning spaces

Setting the

workspace

Setting the cconcept

of the head office

Setting the

entrance area

setting the technical

studies concept

Setting the

workspace

allocating spaces according to their functionality

\section{JG}

Categorizing space adjacencies according to the entrance area

Categorizing spaces according to their public vs. private character

organizing spaces

around the

meeting rooms

organizing the program to separate activities

Fig. 6. The sequence of design ideas associated with the critical moves. 
In the case of the NSSX group three linkographs (architects $\mathrm{AH}, \mathrm{OO}$, and $\mathrm{JG}$ ) render a concentration of design ideas in the beginning of the design session. By contrast, in the same group, LC seems to concentrate his ideas more at the end of the session, which means that he has explored various amounts of ideas before he chose the ideas that fit more with his understanding of the design problem and set them to application. AB seems to record a different sort of design activity as he starts with a set of unrelated design acts aiming to understand the design situation and then passes through an intense design activity producing many ideas in the middle of the session. At the end of his design session he produces fewer new ideas and refers back to the ideas he made in the middle of the session. KS seems to have three main chunks of clustering at the beginning, middle, and end of his design session. However, the progress of KS design ideas in Figure 6 indicates that he started with one main design idea and spent the rest of the session applying it.

For the most part, the design performances in the SSX group spread their design ideas throughout the session, particularly in the case of PE, which suggests a productive form of design activity characterizing all the design sessions. This character also marks some variation and diversity in the design ideas of all the participants. There are some particularities though which take place in the case of BC and CR cases. These particularities can be noticed in the two distinguished layers of concentrated clusters. One of these layers seems to have global attributes as it indicates to patches of ideas linking far-distanced design moves, and the other is more local and links closer design moves. This might mean that the two participants had two layers of creative activity that characterize their design process structures; one layer is global influencing larger portions of the design process and the other is local affecting the sequential design moves. The two layers appear differently in both cases. In BC's case the global layer appears in the beginning of the session and casts effect on the rest of the session. In CR's case the global layer is spread all over the session and has medium linkage length. A similar observable incident to CR's appears in AB's case in the NSSX group. In general, the difference in the number of dark clusters is relative to the amount of design moves in each session and the length of these sessions.

\subsection{Macroscopic cognitive coding of the linkographs}

By coding the design moves in all linkographs according to the macroscopic categorization of Suwa et. al. (1998; see Table 3) we can obtain a combined model of linkography and macroscopic analysis. We can also find out which cognitive actions have more forelinks or backlinks as well as the total number of moves coded by the type of cognitive actions associated with them (see Fig. 7). The amount of functional moves as well as their backlinks and forelinks is relatively larger in the case of the SSX group compared to the NSSX group. This may mean that moves of a functional nature are more dominant and maybe more productive in the SSX group. This functional nature actually accounts for all what is related to the first design filter of Hillier's (1996), including considerations regarding the distribution of occupational spaces and the setting of a circulation system in the layout. Some exceptions appear to be in CR's and JG's cases from each group. CR has a considerably larger number of perception-based design moves with more backlinks and forelinks compared to other members in her group. By contrast JG has less conceptual and perceptual design moves compared to the other members in her group. Therefore, her functional design moves are more frequent and have more effect on the rest of the design process. Moreover, the majority of the critical moves $\mathrm{CM}^{8}$ are functional in the SSX group apart from CR's case, who has a considerable number of perceptionrelated critical moves. The NSSX group has, in general, more critical moves of a perceptual and conceptual nature compared to the SSX group. Apart from the minor exceptions and on the whole, these results correspond to our hypothesis as the SSX group is more functionally oriented than the NSSX group and depends upon the explicit knowledge of the spatial configurations rather than the perceptual or aesthetical considerations in reasoning about their design decisions.

\section{DESIGN OUTCOMES ANALYSIS}

This part of the analysis is mainly concerned with a quantitative evaluation of the final design outcomes produced by the architects in this experiment. The quantitative analysis will use space syntax measures to calculate the spatial and visual configurations in the layouts proposed by the architects. According to space syntax, social organization is embedded in the spatial structure of space. Normally the treelike structure of space reflects a deep and controlled spatial structure and a hierarchy in the social organization. Conversely, the provision of interconnected rings of movement in a layout offers choices for movement routes reducing the depth of space. Spatial relations between adjacent spaces in a layout can be represented using the descriptive methods of justified graphs first presented by Hillier and Hanson (1984). This technique models the spatial network of convex spaces representing each convex space with a circle and each permeable connection between spaces with a line as in Figure 8. The justified graph might be deep or shallow and takes the behavior of branching trees or looping rings. The relation between spaces might be "symmetrical" if, for example, A connects to B = B connects to A. Otherwise, the relation is "asymmetrical." The total amount of asymmetry in a plan from any point relates to its mean depth from that point, measured by its "relative asymmetry" (RA). Spaces that are, in sum, topologically "closest" to all spaces (low RA) are the most shallow in the spatial network and are therefore termed the most integrated. They characteristically have dense pedestrian movement through them. Those that are deepest (high RA) are termed the most segregated. Integration and segregation are global attributes of the spatial network system. Figure 9 shows a typical justified graph. In this graph Hillier (1996) differenti- 


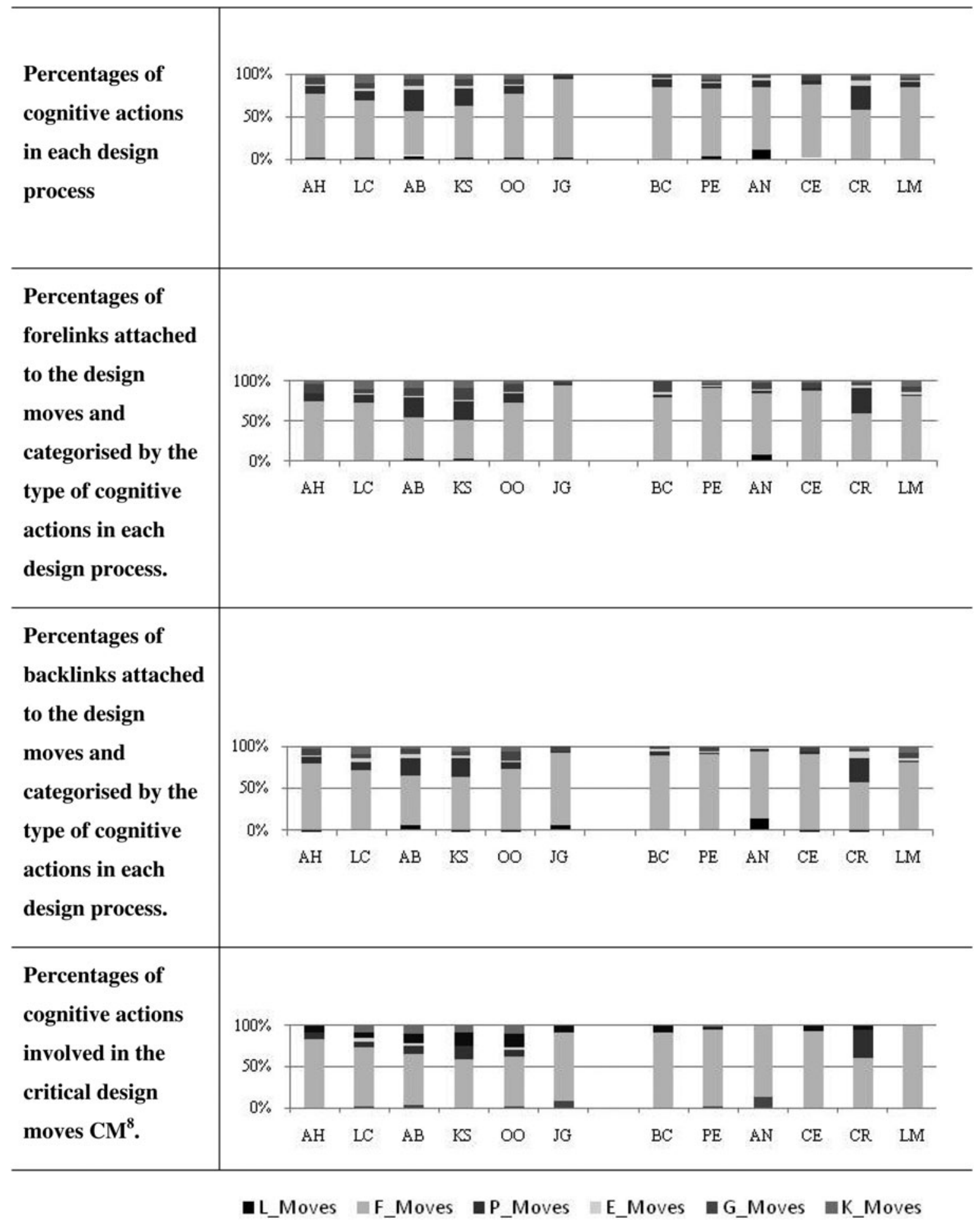

Fig. 7. Different percentage values in relation to the cognitive coding of the linkographs.

ates between four types of spaces: a-types are characterized as dead-end spaces. They connect to no more than one space in the graph. B-types connect to two or more spaces in the graph. However, b-types should not be part of any ring. Conversely, c-types are positioned on one ring. D-type spaces must be in a joint lo- cation between two or more rings. The positioning of these types of spaces within the local and global configurations of the whole network can determine the maximization and minimization of depth in a spatial complex. The increase of a-type locally and d-type globally minimizes depth creating an integrated system, 


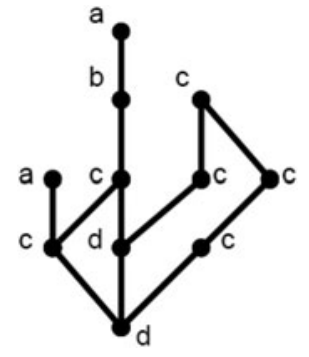

Fig. 8. The types of spatial relations in a justified graph. Adapted from B. Hillier, Space Is the Machine, 1996, p. 249. Cambridge: Cambridge University Press. Copyright 1996 Bill Hillier. Adapted with permission.

whereas the increase of b-type globally and c-type locally maximizes depth resulting with a segregated system.

Convex integration correlates with a building user's static or "occupational" behavior in a building. Convex spaces are spaces where all contained locations are mutually visible from one another. The convex breakup map is formed by identifying the fewest and fattest convex spaces; the fewest to be prevailed. The grayscale colors of the convex map are distributed in five bands according to their integration values. Higher values of convex integration are represented in darker grades of gray and lower values are filled with lighter degrees of gray. Within the network of convex spaces there are different types of spaces: some are more suitable for occupation and more likely to have a larger degree of convexity in their proportions, others allow for denser through-movement because of their elongated shape. Some spaces may even contain both movement and occupational functions. Similar to spatial integration, visual integration is calculated by considering the grid fragments as interconnected convex spaces. It was first introduced by Turner et al. (2001). Considering a grayscale representation of the visibility graph analysis, higher values of visual integration are represented in darker colors whereas lower values are represented with brighter colors.

Overall the summary of the J-graphs shown in Figure 10 with some graphical examples of them illustrated in Figure 9 indicate that the percentages of d-type spaces are higher in the SSX group compared to the NSSX group and conversely for the c-type spaces. This is reflected in a statistical trend indicating that the rings in the spatial network of the designs are more likely to be interconnected in the SSX group and more likely to be separated in the NSSX group: a larger difference between the two groups is observed for the c-type spaces $(t=1.596, p<0.071$, one tailed), whereas the corresponding test for d-type spaces did not approach statistical significance. Given the relatively small sample size and the substantial interindividual differences within each group, these quantitative results by themselves clearly must be interpreted with some caution. However, the spatial depth observed in the J-graphs (see Fig. 9) supports the idea that the designs of the SSX group tend to minimize spatial or topological depth. In addition, it can be noticed that a high count of a-type spaces exist in all the graphs. This might suggest that there are many dead-end spaces in all the proposals marked solely as spaces of occupation. Most of the architects placed the design teams as the main function within the most integrated areas as shown in Figure 11. However, the NSSX group produced different patterns where the corridors are the most integrated spaces within the layout. It can be noticed from the justified graphs examples in Figure 9 that the design teams were shallower to the main entrance; between one or two steps away, in the SSX group. In contrast, the majority of NSSX proposals had the design teams deeper in the organization in relation to the main entrance. In all the proposals the design teams were of c- or d-types, located on one or two rings, although they were in a shallower position in the SSX group than in the NSSX group in relation to the main entrance. The design proposals made by the SSX group segregated the head office so that it was between two and five steps deep from the public access and was usually a dead-end space. This was less evident in the NSSX proposals. In general, the example in Figure 11 demonstrates that the SSX group was able to integrate a larger area in the layout than the NSSX group.

In terms of visual properties of space, it is evident that the SSX group was more successful in creating visually inte-

\section{KS_NSSX architects $\quad 0$ Design team spaces $\quad$ CE_ SSX architects}

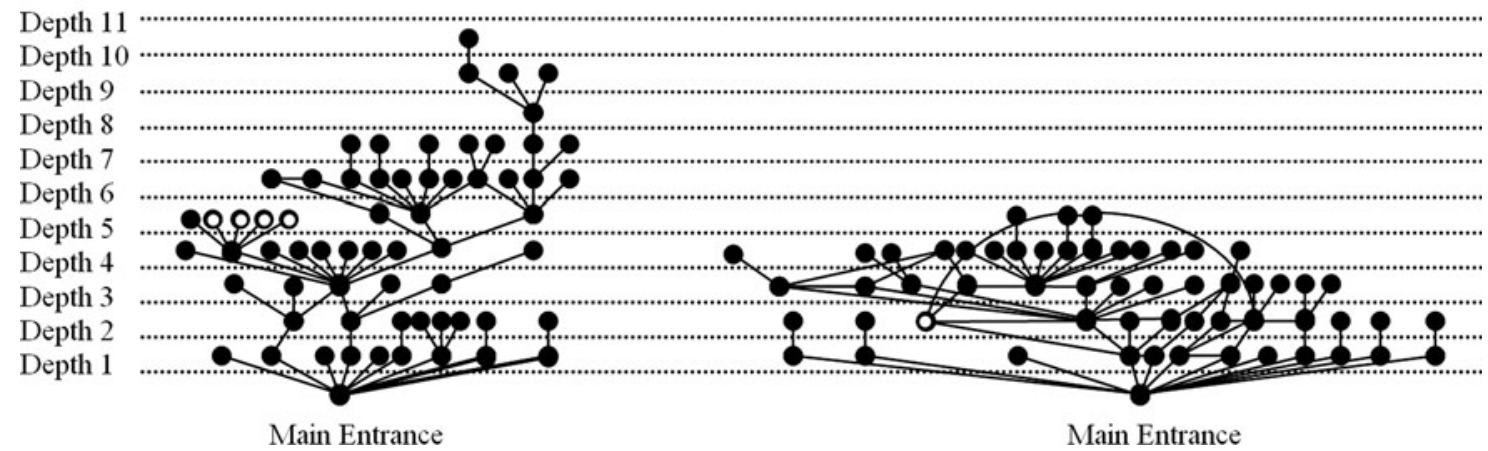

Fig. 9. An example of two J-graphs representing design proposals made by two participants: one is CE from the SSX group and the other is KS from the NSSX group. All J-graphs start from the public entrance. 


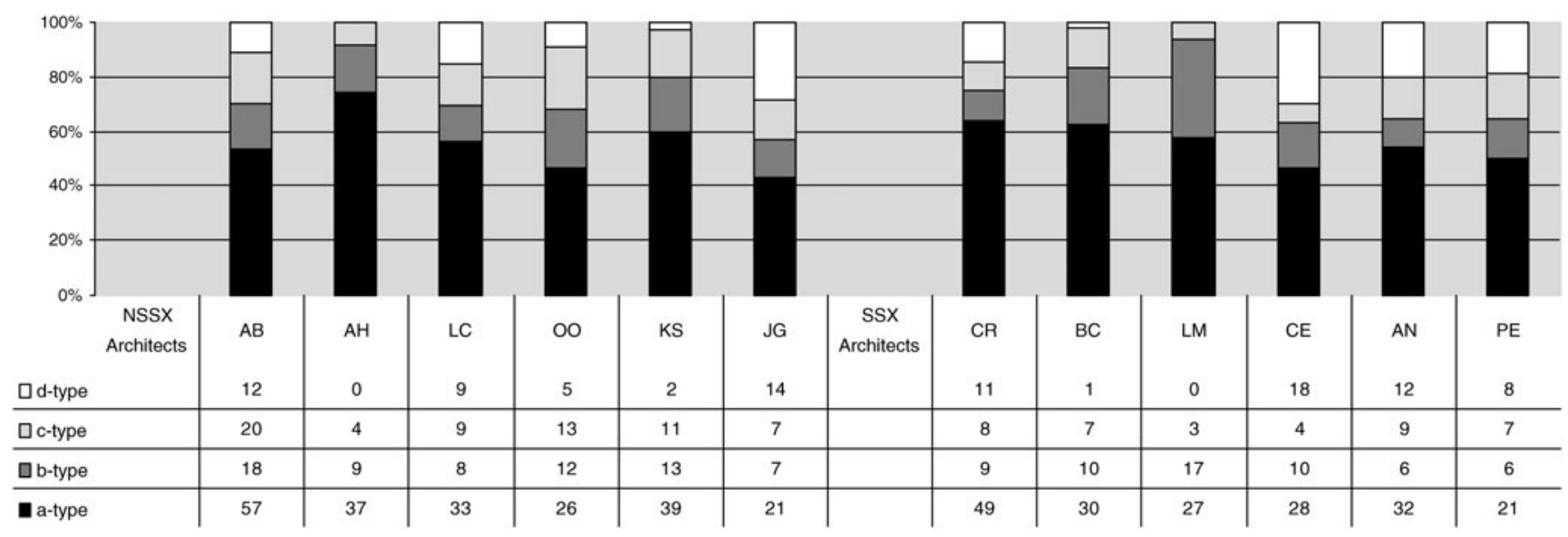

Fig. 10. Percentages and numbers of the four types of spaces in the J-graphs.

grated spaces than the NSSX group. The latter created more fragmented designs and more clustered spaces that have potentials to be merely occupational spaces. Some examples of the spatial and visual analysis are represented in Figure 11. The analysis may suggest that the large, integrated spaces proposed by the SSX group are more likely to encourage broader interactive interfaces between spaces for movement and spaces for occupation promoting more social copresence than the other occupation-oriented designs of the NSSX group.

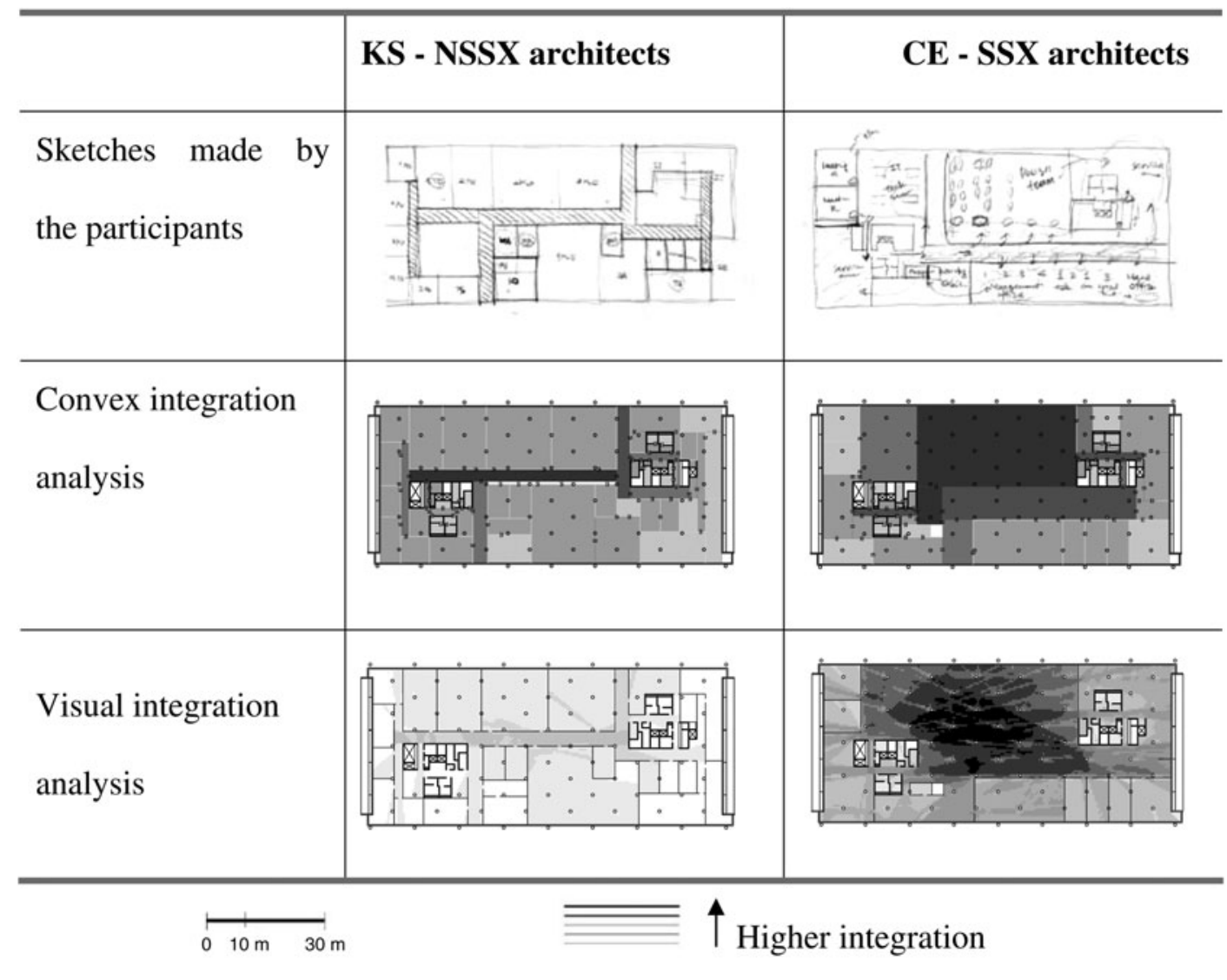

Fig. 11. Spatial analysis of the design proposals using space syntax tools (Turner, 2006). Convex integration analysis shows the degree of integration between the convex spaces in the design proposals. Visual integration analysis shows the degree of visual integration between each point in the layout grid. It takes into account all of the visible points in a layout without considering their accessibility. Thus, low or transparent partitions are not considered. 


\section{DISCUSSION}

The verbal and visual data collected throughout the design experiments have revealed some differences and similarities between the two groups in addition to considerable individual differences between the participants. The cognitive and spatial analyses have also indicated some commonalities and revealed some differences between the two groups.

The verbal transcripts of the design process highlighted some similarities between the different design approaches. The design process similarities appeared in the reactions of designers when faced with the design problem; most of them stressed their own vision for an open plan layout against the normative brief demands for individual spaces. Most of them started their design process by calculating the number of space users, and were concerned about the spaces areas, the scale, and the structural system. Both groups raised the issue of visibility frequently. Most of them designed the circulation from the point of view of space users and their potential spatial experiences. They often started by defining public and private access points, followed by allocating spaces accordingly. Apart from KS, who was more interested in satisfying lighting requirements, all the participants started their designs by attempting to understand the organizational structure, sometimes emphasizing and sometimes avoiding the hierarchical structure of the organization, and this was evidenced in their subsequent spatial solutions. All of the participants were preoccupied with the idea of open plan layout, although most of the NSSX participants have ended up limiting the visual qualities of their solutions by proposing opaque partitions. Verbal comments of the SSX group revealed their knowledge of space syntax, especially in PE's proposal, who avoided creating large enclosed spaces in order not to "block potential movement." CE and LM talked about the firm's Directors usually being segregated in the spatial structure. Similar comments were more implicit rather than explicit in the NSSX group's verbal transcripts, such as the segregation of Directors in "boxes" that OO stressed in his design, or the vi- sual experience of space that $\mathrm{LC}$ and $\mathrm{AB}$ repeatedly referred to in their designs.

The results generally indicate that the SSX group was more confident in decision making, although it is not unequivocally clear if both groups were expressing their thoughts discursively; however, the method used in semantic recording during the design tasks is still probably the best method available for making these thoughts explicit during design actions. The short time needed by the SSX group for the design tasks may support the interpretation that this group felt more confident in solving the design problem than the NSSX group. On average, the number of original ideas produced by the SSX participants during the course of their design processes is relatively higher than in the NSSX group (see Fig. 12). This result is supported if we consider that the time consumed for problem solving as well as the number of design moves are less in the SSX case than in the NSSX case. In particular, CR seems to have produced a considerably high amount of original design ideas within a relatively short amount of time (15 min).

In considering the protocol analysis SSX architects were proved, on average, to perform slightly better than NSSX architects. The link index and $\mathrm{CM}^{8}$ provided evidence for more productive activity in SSX performance particularly in the cases of PE, CE, and BC, whereas AN and LM has a particularly low productive performance. The variations between the performances of SSX participants were more pronounced than the ones in NSSX group. In two cases, LC and OO seem to have relatively high productive performance within their group. In other two cases, $\mathrm{KS}$ and $\mathrm{AB}$ are less productive than the rest of their group. The designs produced by these participants reflect corresponding effects as the architects with the most productive performance produced spatially and visually well-integrated spaces. By contrast, designs produced by architects with low productive performance were characterized as more spatially and visually segregated (as evidenced by a moderate, but substantial correlation of 0.45 between visual integration and link index and 0.40 between

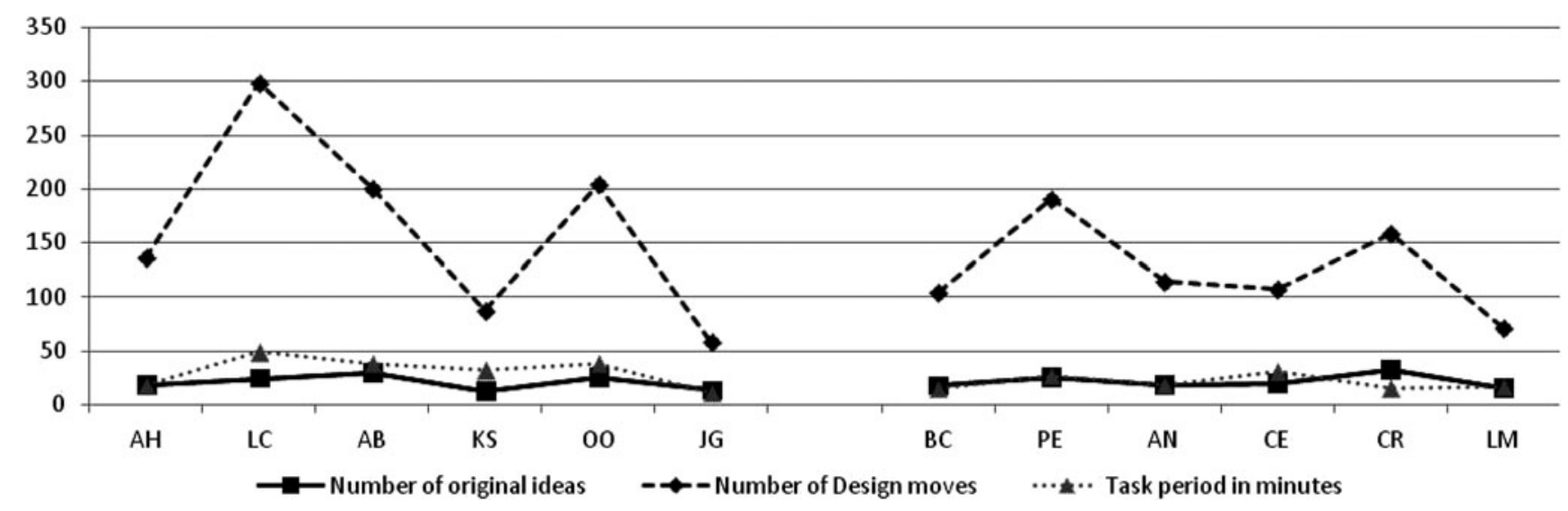

Fig. 12. The number of original ideas produced by the participants plotted against the number of design moves and the period of time the participants required to solve the design task. 
visual integration and $\mathrm{CM}^{8}$ ). In general, productive design performance and well-integrated solutions were more evident in SSX group than in NSSX group.

The general distribution of nodes in the linkographs showed that all participants produced ideas at every stage in their design processes, but there were some differences between the lengths of the links between both groups. These differences can be interpreted in the form of shorter links in SSX linkographs compared with longer ones in NSSX linkographs. This became clearer with introduction of the clustering analysis revealing more variety in the clustering of nodes in NSSX linkographs compared with the ones of the SSX group. Spots of concentrated clusters appeared at the beginning of three linkographs: AH, OO, and JG. This means that as the design process started, idea generation was more intense. LC had a contrasting behavior as his design ideas seem to converge at the very end of the design process. Although AB produced more ideas in the middle of the session, ideas of $\mathrm{KS}$ were distributed throughout his design process. Idea generation in the SSX group was a more continual and steady process throughout the duration of the design processes. This points to productive and diverse creative activity in the SSX group. Some particular models in the form of a pair of global and local layers can be detected in BC and CR cases: a global layer linking far-distanced design moves and a local one connecting adjacent design moves. Their creative activity is characterized as a process of interchange and interaction between both layers of thoughts: one connecting the process as whole and the other connecting sequential design decisions. The global layer in BC's case appears to dominate in the start of the design process, whereas in CR's case it is dominant throughout the duration of the design task.

It might be important to point out that $\mathrm{CR}$ has the longest experience using space syntax methodology, and she generated the most spatially and visually integrated layout compared to all other participants. CR also produced a relatively large amount of original design ideas. Yet, she was also unique among her group by having a high amount of perceptual-based design moves in a manner more similar to the NSSX participants. The rest of the SSX group demonstrated a high number of design moves, including high count of critical moves characterized by their functional nature. This regularity is less apparent in the NSSX group as the perceptual and conceptual moves rise in numbers and reduce the dominant effect of functional moves. This may indicate that the SSX group were more functionally oriented in their designs compared with the NSSX group. An indication of that can be found in their designs as the circulation systems they have produced proved to optimize depth producing a shallower spatial structure compared with the NSSX group.

In general, the quantitative analysis of spatial design outcomes emphasized the idea that the SSX proposals were inclined to minimize depth and promote social copresence in office environments, with wide visual view and well-integrated spaces for teams working together. The spaces produced by SSX group were intelligible and interconnected with rings linking the two cores. The NSSX group often produced disconnected localized rings, organizing spaces in a treelike hierarchy from the entrances to the corridors.

\section{CONCLUSIONS}

The protocol analysis of design processes as well as the evaluations of spatial and visual configurations presented here support our hypothesis. In other words, the explicit knowledge of spatial configurations does appear to positively influence architects in solving design problems intuitively to some extent. By means of explicit spatial knowledge, architects produce relatively better design outcomes through rendering the nondiscursive techniques of problem solving in order to become more discursive. Such a result is subject to the limitations of the design task circumstances and the method in which the intuitive design processes were modeled. In general, it can be concluded from the analysis that the SSX architects were more able to express their thoughts explicitly compared with the NSSX group. The explicit knowledge of spatial configurations that the SSX group possesses might have helped them in being more confident, productive, and original in their design processes and might have helped guide them to provide more integrated solutions than the NSSX group. This is most obvious in the performances of $\mathrm{CR}, \mathrm{PE}, \mathrm{BC}$, and $\mathrm{CE}$. It becomes evident in the verbal transcripts that all of the participants in both groups mentioned the same issues regarding the accessibility and visual connectivity of spaces. Their design outcomes reflect the optimization process they have gone through in order to approach possible solutions, particularly in terms of the first set of filters constraining their ideas in order to allow for the basic spatial functionality in the layout.

Their views were mostly user based. In their verbal comments most of them seem to have thought about spatial arrangement and related that to how hypothetical users would circulate, use spaces, and communicate with each other according to their job nature. There were some different concerns expressed by NSSX participants as they have repeatedly referred to privacy issues and confidentiality, and they have reflected that in clusterlike designs. There are regularities in the designer's considerations in general, as all of them appeared to concentrate mostly on permeability issues. The participants have frequently compared their decisions to their previous experiences working in similar environments, a typical example of case-based reasoning in architectural design (Goel \& Pirolli, 1992; Maher et al., 1995). Yet, conceptual design moves including knowledge retrieval ones were more frequent in the NSSX group than in the SSX group. Similar to that, perceptual moves appeared to be more common in the NSSX group but functional moves were more dominant in the SSX group. This might indicate that NSSX participants were more preoccupied by surface features or aesthetic appearances in their design, while SSX participants were more discursive, using logic and rule-based judgment in 
their design decisions in order to satisfy functional requirements as their main priority.

The design solutions reflect more integrated spatial structures in the SSX proposals than in the NSSX designs. This result is quite remarkable because it supports the suggestion that by exposing the spatial configurations of space to an explicit model of description it could be possible to enhance the design processes and design outcomes of architects. This knowledge of space syntax, if employed in the evaluative process of reasoning during design, thus has a positive impact on the architect's performance and design solutions, and it can reduce the time consumed for problem solving. It is important to emphasize that this result is only viable in terms of the first filter in a design process that is almost exclusively about spatial configurations. Other methods of evaluation could be accommodated by extending the current study to include an integrated model of evaluation. This depends on how feasible it is to quantify other evaluation rationales to narrow the set of solutions in an approach to reach the solution that best corresponds to the design problem. An integrated model of evaluation might be reflected in an artificial intelligence model that simulates design creativity based on discursive problem solving techniques and explicit knowledge resources.

This research has only explored the primary use of spatial configurations as the first set of constraints to optimize a design process. This is mainly because of the availability of scientific and observation-based research in this area that provides reliable quantification measures of this particular method of spatial evaluation. Nonetheless, future research will consider observing the drawing process itself to track the mechanism of reasoning and its consequential representations. It would also be imperative to expand the number of participants and present improved control measures when selecting them to obtain more consistent results. Increasing the number of participants will help generalize the findings and present results that tend to be more statistically significant. Attempts might also be made to present some variations in the design brief, as well as probably testing architectural design problems of a different nature to reinforce the findings by changing the mode and circumstances of the experiment.

\section{ACKNOWLEDGMENTS}

This paper reports a substantially extended analysis on a previously published study (Al-Sayed et al., 2008). We are very grateful to the researchers and staff at Space Syntax Ltd and the Bartlett School of Graduate Studies, University College London, for providing valuable advice and insights that had positive impact on our research. Our greatest gratitude goes to the architects who participated in our design experiment for their patience and the valuable time they spent solving the design tasks. We gratefully acknowledge the financial support of Damascus University, University College London, for its contribution, and the German Research Council DFG that cofunded this research within the SFB/TR8 Spatial Cognition Project R6-SpaceGuide.

\section{REFERENCES}

Al-Sayed, K., Conroy Dalton, R., \& Hölscher, C. (2008). Discursive and nondiscursive design processes. Proc. 3rd Int. Conf. Design Computing and Cognition (Gero, J.S., \& Goel, A.K., Eds.), pp. 635-654. Amsterdam: Springer.

Bertel, S., Vrachliotis, G., \& Freksa, C. (2007). Aspect-oriented building design: toward computer-aided approaches to solving spatial constraints in architecture. In Applied Spatial Cognition: From Research to Cognitive Technology (Allen, G.L., Ed.), pp. 75-102. Mahwah, NJ: Erlbaum.

Boden, M. (1994). Dimensions of Creativity. London: MIT Press.

Carbonell, J.G. (1983). Learning by analogy: formulation and generalizing plans from past experience. In Machine Learning: An Artificial Intelligence Approach (Michalski, R.S., Carbonell, J.G., \& Mitchell, T.M., Eds). Palo Alto, CA: Tioga Publishing Company.

Carbonell, J.G. (1986). Derivational analogy: a theory of reconstructive problem solving and expertise acquisition. In Machine Learning: An Artificial Intelligence Approach (Michalski, R.S., Carbonell, J.G., \& Mitchell, T.M., Eds.). Palo Alto, CA: Tioga Publishing Company.

Carrara, G., \& Fioravanti, A. (2005). Creative architectural design boosted by itc enhanced incubator: how to facilitate tighter integrated collaboration to boost the emergence and diffusion of creativity. Proc. HI'05, 6th Int. Conf. Computational and Cognitive Models of Creative Design (Gero, J.S., \& Maher, M.L., Eds.), pp. 275-300. Sydney: University of Sydney.

Dienes, Z., \& Perner, J. (1999). A theory of implicit and explicit knowledge. Behavioral and Brain Sciences 22(5), 735-808.

Ericsson, K.A., \& Simon, H.A. (1993). Protocol Analysis: Verbal Reports as Data. Cambridge, MA: MIT Press.

Goel, V. (1995). Sketches of Thought. Cambridge, MA: MIT Press.

Goel, V., \& Pirolli, P. (1992). The structure of design problem spaces. Cognitive Science 16, 395-429.

Goldschmidt, G. (1990). Linkography: assessing design productivity. In Cyberbetics and System (Trappl, R., Ed.), pp. 291-298. Singapore: World Scientific.

Goldschmidt, G. (1992). Criteria for design evaluation: a process-oriented paradigm. In Evaluating and Predicting Design Performance (Kalay, Y.E., Ed.), pp. 67-79. New York: Wiley.

Goldschmidt, G. (1995). The designer as a team of one. Design Studies 16(2), 189-209.

Goldschmidt, G. (2001). Visual analogy—a strategy for design reasoning and learning. In Design Knowing and Learning: Cognition in Design Education (Eastman, C., McCracken, M., \& Newstetter, W., Eds.), pp. 199-219. New York: Elsevier.

Hillier, B. (1996). Space Is the Machine. Cambridge: Cambridge University Press.

Hillier, B., \& Hanson, J. (1984). The Social Logic of Space. Cambridge: Cambridge University Press.

Kan, W.T., \& Gero, J.S. (2008). Acquiring information from linkography in protocol studies of designing. Design Studies 29(4), 315-337.

Kryssanov, V.V., Tamaki, H., \& Kitamura, S. (2001). Understanding design fundamentals: How synthesis and analysis drive creativity, resulting in emergence. Artificial Intelligence in Engineering 15(4), 329-342.

Maher, M.L., Balachandran, M.B., \& Zhang, D.M. (1995). Case-Based Reasoning in Design. Hillsdale, NJ: Erlbaum.

Oxman, R. (1990). Prior knowledge in design: a dynamic knowledge-based model of design and creativity. Design Studies 11(1), 17-28.

Oxman, R. (1997). Design by re-representation: a model of visual reasoning in design. Design Studies 18(4), 329-347.

Pahl, G., \& Beitz, W. (1996). Engineering Design: A Systematic Approach, 2nd ed. London: Springer-Verlag.

Shpuza, E. (2006). Floorplate shapes and office layouts: a model of the effect of floorplate shape on circulation integration. $\mathrm{PhD}$ Thesis. Georgia Institute of Technology.

Simon, H.A. (1984). The structure of ill-structured problems. In Developments in Design Methodology (Cross, N., Ed.), pp. 317-327. New York: Wiley.

Suwa, M., Purcell, T., \& Gero, J. (1998). Macroscopic analysis of design processes based on a scheme for coding designer's cognitive actions. Design Studies 19(4), 455-483.

Turner, A. (2006). UCL Depthmap: Spatial Network Analysis Software, Version 6.0818b. London: University College London, VR Centre of the Built Environment.

Turner, A., Doxa, M., O’Sullivan, D., \& Penn, A. (2001). From isovists to visibility graphs: a methodology for the analysis of architectural space. Environment and Planning B: Planning and Design 28(1), 103-121. 
Kinda Al-Sayed is currently tutoring the Applied Architectural Research Module for the Master's program in AAC, Bartlett School of Graduate Studies, University College London. She is also a research student at Bartlett. She has worked as a Teaching Fellow at Damascus University and is an architect with several years of practice experience. Her main interests are architectural design and cognition, intelligent design models, and computational models of urban growth.

Ruth Conroy Dalton is a Lecturer in architectural morphology and theory at Bartlett School of Graduate Studies, University College London. She directs the Theory in Architecture and Urban Space Module for the Master's program in AAS. She is a chartered architect with many years of practice experience. Her research interests include spatial navigation and cognition, visibility field analyses or measures, and virtual environments or virtual social spaces.

Christoph Hölscher is an Assistant Professor at the Center for Cognitive Science, University of Freiburg, Germany. After graduating in psychology, Christoph worked as a consultant and project leader in the software industry, specializing in user modeling and usability. Since returning to academia, he has focused on interdisciplinary practice among cognitive science, environmental psychology, and architecture, undertaking research in human navigation and orientation through buildings as well as in the cognitive processes of architects while in the process of designing. Dr. Hölscher is a member of the board of directors of the Transregional Collaborative Research Center SFB/TR8 Spatial Cognition and an Honorary Senior Research Fellow at the Bartlett School of Architecture, University College London. 